\section{RSP}

http://www.rsp.fsp.usp.br/
Revista de Saúde Pública

\title{
Análise de possíveis fatores de interferência no uso da voz durante atividade docente
}

Bárbara Gabriela Silva', Tiago Visacre Chammas', Marcia Simões Zenari', Renata Rodrigues Moreira", Alessandra Giannella Samelli', Kátia Nemr'

' Universidade de São Paulo. Faculdade de Medicina. Departamento de Fisioterapia, Fonoaudiologia e Terapia Ocupacional. São Paulo, SP, Brasil

" Universidade de São Paulo. Hospital Universitário. São Paulo, SP, Brasil

\section{RESUMO}

OBJETIVO: Mensurar o risco de disfonia em professores, bem como investigar se os aspectos vocais perceptivo-auditivos e acústicos em situação de ruído, a relação sinal-ruído e os níveis de ruído em sala de aula estão associados à presença de disfonia.

MÉTODOS: Pesquisa transversal observacional com 23 professores da educação infantil e ensino fundamental de uma escola particular do município de São Paulo divididos nos grupos sem disfonia e com disfonia. Foram realizados os seguintes procedimentos: protocolo de rastreio de risco de disfonia geral (PRRD-Geral) e complementar para voz falada - professor (PRRD-Específico), gravação da voz durante aula e em situação individual em sala silenciosa, medição da relação sinal-ruído e dos níveis de ruído das salas de aula.

RESULTADOS: Foram encontradas diferenças entre os grupos quanto à atividade física (PRRD-Geral) e particularidades da profissão (PRRD-Específico), bem como em todos os aspectos da análise vocal perceptivo-auditiva. No grupo sem disfonia, foram encontrados sinais de desgaste da voz. Quanto aos recursos vocais nas situações de ruído e silêncio, identificamos diferença para produção de ataque vocal brusco e tendência de uma fala mais precisa na situação-ruído. Tanto a relação sinal-ruído quanto os níveis de ruído das salas durante a aula foram elevados nos dois grupos.

CONCLUSÕES: Os professores dos dois grupos estão expostos a riscos elevados para o desenvolvimento de disfonias e apresentam sinais vocais negativos em maior ou menor grau. A relação sinal-ruído apresentou-se inadequada em grande parte das salas de aula, considerando-se as normas tanto para crianças com audição normal quanto para aquelas com perda auditiva, assim como os níveis de ruído equivalentes.

DESCRITORES: Professores Escolares. Distúrbios da Voz, epidemiologia. Ruído, efeitos adversos.

Como citar: Silva BG

Chammas TV, Zenari MS, Moreira RR, Samelli AG, Nemr K. Análise de possíveis fatores de interferência no uso da voz durante atividade docente. Rev Saude Publica. 2017;51:124.

Copyright: Este é um artigo de acesso aberto distribuído sob os termos da Licença de Atribuição Creative Commons, que permite uso irrestrito, distribuição e reprodução em qualquer meio desde que o autor e a fonte originais sejam creditados.

\section{Perda Auditiva. Fatores de Risco. Condições de Trabalho.}




\section{INTRODUÇÃO}

Estudos sobre a voz dos professores demonstram elevado índice de disfonia nessa classe profissional ${ }^{3,10,24}$. A gravidade da disfonia pode interferir negativamente na viabilização do trabalho docente, com consequente prejuízo para a compreensão dos alunos ${ }^{22} \mathrm{e}$, em casos mais graves, afastamento desses profissionais de suas funções ${ }^{19}$.

Dentre as condições de trabalho que permeiam o processo saúde-doença dos docentes, destacam-se as longas jornadas de trabalho, a utilização vocal prolongada e excessiva ${ }^{5}$, bem como os níveis elevados e constantes de ruído nas salas de aula ${ }^{11}$, acima do recomendado pela NBR 10152, entre $40 \mathrm{~dB}$ e $50 \mathrm{~dB}(\mathrm{~A})^{\mathrm{a}}$.

Durante as aulas, o ruído excessivo, além de interferir na atenção e concentração dos docentes ${ }^{11}$ e dificultar o processamento auditivo das informações essenciais para o desenvolvimento das atividades, transfigura-se em um estímulo sonoro competitivo à produção vocal, podendo gerar ajustes inadequados da voz, elevando o risco de disfonia ${ }^{20,24}$.

Um fator acústico de interferência em sala de aula, e importante ferramenta para monitorar tanto o ruído quanto as vozes dos professores, consiste na relação sinal-ruído $(\mathrm{RSR})^{4}$, calculada a partir da diferença, em decibels (dB), entre o nível do sinal de interesse (no caso, a voz do professor) e a intensidade do ruído de fundo do ambiente; pode variar de acordo com a distância e intensidade do sinal de interesse, a reverberação do ambiente e o ruído de fundo 8 .

A investigação, tanto do risco de disfonia quanto do ruído durante a atividade docente, tem o propósito de auxiliar no direcionamento de programas e atividades de promoção e prevenção de distúrbios vocais, bem como nas modificações necessárias na organização e condições de trabalho para, não só diminuir o número de afastamentos dos professores do trabalho, mas melhorar o ensino e a qualidade de vida dos envolvidos.

O objetivo deste estudo foi mensurar o risco de disfonia em professores, bem como investigar se os aspectos vocais perceptivo-auditivos e acústicos em situação de ruído, a relação sinal-ruído e os níveis de ruído em sala de aula estão associados à presença de disfonia.

a Associação Brasileira de Normas Técnicas. NBR 10152: níveis de ruído para conforto acústico. Rio de Janeiro: ABNT; 2000

${ }^{\text {b }}$ Sampaio AP, Simões-Zenari M, Nemr NK. Aplicabilidade de protocolo de rastreio de risco de disfonia em professores. São Paulo: Faculdade de Medicina da Universidade de São Paulo; 2015. Trabalho de Conclusão de Curso de Fonoaudiologia.

cAmerican Speech-LanguageHearing Association, ASHA Division 3 - Voice and Voice Disorders; University of Pittisburg, Department of Communication Science and Disorders. A Consensus Auditory - Perceptual Evaluation of Voice (CAPE -V). Pittisburgh: ASHA; 2002 [citado 23 out 2017]. Disponível em: https://csd.wisc. edu/slpgames/capev_activities/ capev_Instructions.pdf

\section{MÉTODOS}

Pesquisa transversal observacional aprovada pelo Comitê de Ética em Pesquisa da Faculdade de Medicina da Universidade de São Paulo (Parecer 169/15), realizada em uma escola de ensino infantil e fundamental particular do município de São Paulo.

Vinte e três professoras, com média de idade de 37,26 anos $(\mathrm{DP}=7,0)$, assinaram o termo de consentimento livre e esclarecido.

Na própria escola, foram realizados os seguintes procedimentos:

- Aplicação do Protocolo de Rastreio de Risco de Disfonia Geral (PRRD-Geral) $)^{15}$ e Protocolo de Rastreio de Risco de Disfonia Complementar para Profissional da Voz Falada, Professor (PRRD-Específico) ${ }^{b}$, para identificação de queixas vocais, hábitos de vida geral e ocupacionais, entre outros. Os protocolos foram explicados pelos pesquisadores e preenchidos pelos professores na presença dos mesmos.

- Gravação da voz em ambiente silencioso (situação-silêncio) para posterior análise vocal perceptivo-auditiva e acústica: vogais sustentadas, frases e fala espontânea de acordo com o protocolo CAPE-V $\mathrm{V}^{\mathrm{c}}$. Foi utilizado o equipamento ZOOM H4 posicionado perpendicularmente a $30 \mathrm{~cm}$ da boca do professor.

- Gravação da voz com o gravador ZOOM H4 posicionado no meio da sala de aula (situação-ruído) durante 15 minutos de fala efetiva (situação de aula expositiva). 
- Simultaneamente à gravação dos professores nas salas de aula, utilizou-se um dosímetro, SVANTEK (modelo SV102, Polônia), posicionado no centro da sala, para medição dos níveis de pressão sonora (níveis máximo, mínimo e ruído equivalente [Leq]), durante 15 minutos.

\section{Análise dos Dados}

Foi obtido escore final dos protocolos de rastreio, que consiste na soma dos valores dos escores parciais de cada subitem; no PRRD-Geral, varia de zero a 131 e no PRRD-Específico, de zero a 56 para homens e de zero a 60 para mulheres ${ }^{15}$. Quanto maior o valor obtido, maior o risco de disfonia ${ }^{15, b}$.

A análise perceptivo-auditiva das gravações de voz obtidas no silêncio foi realizada por um juiz fonoaudiólogo especialista em voz com ampla experiência em avaliação com CAPE-V e elevada confiabilidade interna de julgamento, aferida em estudo anterior ${ }^{15}$. A partir do valor do grau geral de alteração vocal, os professores foram divididos em dois grupos: sem disfonia (GSD), para valores entre zero e 34; ou com disfonia (GCD), para valores superiores a $34^{12}$. Procedeu-se a avaliação acústica da mesma amostra por meio do programa de uso livre Praat, considerando-se as medidas automáticas: frequência fundamental (normalidade: $150 \mathrm{~Hz}$ a 250 Hz); jitter, (0,30\% a 0,50\%); shimmer (3\% e 5\%); e proporção harmônico-ruído (22 dB) $)^{2,17, d}$.

As vozes captadas em situação-silêncio e situação-ruído foram analisadas por dois dos pesquisadores por consenso, sendo classificadas quanto aos recursos vocais ${ }^{9}$ : tipo de voz (neutro, alterado); ataque vocal (isocrônico, brusco, aspirado); loudness (forte, médio, fraco); pitch (grave, médio, agudo); ressonância (equilibrada, oral, laringofaríngica, nasal, hiponasal); articulação (precisa, reduzida); velocidade de fala (lenta, média, acelerada); e coordenação pneumofonoarticulatória (presente, ausente).

Com o registro vocal captado em aula, mensurou-se a RSR de cada sala por meio do programa Audacity 2.0.6, protocolo Canadian E-Ramp-Inc. Para classificação da RSR em adequada ou inadequada, foram considerados os valores de corte de $+15 \mathrm{~dB}$ para salas de aula de crianças com audição normal (ASHA, 2000) e de $+20 \mathrm{~dB}$ para crianças com perda auditiva (British Association of Teachers of the Deaf, 2009).

Em relação à medição dos níveis de pressão sonora em sala de aula, para classificação em adequados ou elevados, utilizou-se a NBR 10152 (ABNT, 2000)a , que considera aceitável para salas de aula ruído ambiente de $40 \mathrm{~dB}$ a $50 \mathrm{~dB}(\mathrm{~A})$.

\section{Análise Estatística}

Foram utilizadas medidas descritivas e aplicados os testes ANOVA não pareado e qui-quadrado (teste Exato MID-P) com nível de significância de 0,05.

¿ Leão SHS. Análise espectrográfica acústica de vozes rugosas, soprosas e tensas [dissertação]. São Paulo: Universidade Federal de São Paulo - UNIFESP; 2008.

e Crandell CC, Smaldino J). Classroom acoustics for children with normal hearing and with hearing impairment. Lang Speech Hear Serv Sch. 2000;31(4):362-70. https:// doi.org/10.1044/01611461.3104.362

${ }^{f}$ Glasgow M. Classroom acoustics: recommended standards: classroom acoustic standards for children with sensori-neural hearing loss. Cheshire (UK): The British Association of Teachers of the Deaf; 2009.

\section{RESULTADOS}

Quanto à divisão dos grupos a partir dos valores do Grau Geral do CAPE-V, obteve-se: GSD composto por 11 participantes (média do escore de 20,81, DP = 10,1) e GCD composto por 12 indivíduos (média do escore de 52,91, DP = 12,43).

No que se refere ao PRRD-Geral, encontrou-se diferença apenas para o escore parcial do subitem prática regular de atividade física (Tabela 1), com menor ocorrência no GCD. Para o PPRD-Específico, encontrou-se diferença no subitem especificidades da profissão (Tabela 1), em que o GSD apresentou pontuação mais elevada. Em relação ao escore final do PRRD-Geral e do PRRD-Específico, não houve diferença entre os grupos.

Não foram encontradas diferenças entre os grupos em nenhum dos aspectos vocais acústicos avaliados. Em relação ao CAPE-V, observou-se diferença entre os grupos em todos os aspectos, com maior alteração para o GCD (Tabela 2). 
Tabela 1. Média, desvio padrão, mínimo e máximo referentes aos escores do PRRD-Geral e PRRD-Complementar para os grupos com e sem disfonia.

\begin{tabular}{|c|c|c|c|c|c|c|}
\hline Característica & Grupos & Média & DP & Mínimo & Máximo & $p^{a}$ \\
\hline \multicolumn{7}{|c|}{ PRRD-Geral } \\
\hline \multirow{2}{*}{ Escala analógica visual } & GSD & 3,38 & 2,37 & 0 & 6,3 & \multirow{2}{*}{0,066} \\
\hline & GCD & 5,24 & 2,24 & 1 & 8,3 & \\
\hline \multirow{2}{*}{ Alterações vocais anteriores } & GSD & 1,64 & 0,92 & 0 & 2 & \multirow{2}{*}{0,505} \\
\hline & GCD & 1,83 & 0,39 & 1 & 2 & \\
\hline \multirow{2}{*}{ Sintomas atuais } & GSD & 20 & 13,17 & 2 & 39 & \multirow{2}{*}{1} \\
\hline & GCD & 20,16 & 7,75 & 9 & 33 & \\
\hline \multirow{2}{*}{ Uso vocal fora do trabalho } & GSD & 2,27 & 1,27 & 0 & 4 & \multirow{2}{*}{0,534} \\
\hline & GCD & 2,42 & 0,79 & 1 & 4 & \\
\hline \multirow{2}{*}{ Alimentação } & GSD & 2,36 & 1,36 & 0 & 4 & \multirow{2}{*}{0,578} \\
\hline & GCD & 2,08 & 0,99 & 1 & 4 & \\
\hline \multirow{2}{*}{ Hidratação } & GSD & 1,73 & 1,27 & 0 & 3 & \multirow{2}{*}{0,584} \\
\hline & GCD & 1,42 & 1,38 & 0 & 3 & \\
\hline \multirow{2}{*}{ Medicação } & GSD & 0,09 & 0,3 & 0 & 1 & \multirow{2}{*}{0,336} \\
\hline & GCD & 0,25 & 0,45 & 0 & 1 & \\
\hline \multirow{2}{*}{ Fumo } & GSD & 0,18 & 0,4 & 0 & 1 & \multirow{2}{*}{0,609} \\
\hline & GCD & 0,33 & 0,89 & 0 & 3 & \\
\hline \multirow{2}{*}{ Sono } & GSD & 1,36 & 1,36 & 0 & 3 & \multirow{2}{*}{0,755} \\
\hline & GCD & 1,5 & 0,52 & 1 & 2 & \\
\hline Doencas & GSD & 0,91 & 0,94 & 0 & 2 & 0348 \\
\hline Doenças & GCD & 1,33 & 1,15 & 0 & 3 & 0,340 \\
\hline & GSD & 0,18 & 0,4 & 0 & 1 & \\
\hline Hıstorıco de alteraçao vocal na tamılıa & GCD & 0 & 0 & 0 & 0 & 0,134 \\
\hline & GSD & 0 & 0 & 0 & 0 & \\
\hline Dınamıca tamılıar & GCD & 0,08 & 0,29 & 0 & 0 & 0,351 \\
\hline Atividade fícica & GSD & 0,36 & 0,5 & 0 & 1 & 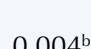 \\
\hline Alividade issica & GCD & 0,92 & 0,29 & 0 & 1 & $0,004^{\circ}$ \\
\hline lazer & GSD & 0 & 0 & 0 & 0 & 0171 \\
\hline Lazer & GCD & 0,17 & 0,39 & 0 & 1 & $0,1 / 1$ \\
\hline Fscore final & GSD & 36,47 & 16,26 & 11 & 62 & 0.572 \\
\hline Escore minal & GCD & 39,74 & 10,75 & 25 & 58 & $0,3 / 2$ \\
\hline & PRRL & omplem & & & & \\
\hline Tempo de uso & GSD & 2,73 & 1,01 & 1 & 4 & 0.596 \\
\hline rempo de uso & GCD & 2,92 & 0,67 & 2 & 4 & 0,390 \\
\hline Proficcão & GSD & 11,72 & 0,47 & 11 & 12 & 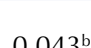 \\
\hline Promssao & GCD & 10,91 & 1,16 & 9 & 12 & $0,043^{\circ}$ \\
\hline Condicões ambientais & GSD & 8,64 & 1,03 & 8 & 11 & 0534 \\
\hline Condiçoes amblentals & GCD & 9,25 & 3,08 & 2 & 14 & 0,534 \\
\hline & GSD & 0,27 & 0,47 & 0 & 1 & \\
\hline Atastamento & GCD & 0,08 & 0,29 & 0 & 1 & 0,249 \\
\hline Fum & GSD & 0 & 0 & 0 & 0 & 0171 \\
\hline rumo & GCD & 0,5 & 1,17 & 0 & 3 & $0,1 / 1$ \\
\hline & GSD & 0,27 & 0,47 & 0 & 1 & 0021 \\
\hline Consumo de alcool & GCD & 0,25 & 0,45 & 0 & 1 & $0,9<1$ \\
\hline Consumo de drogas & GSD & 0 & 0 & 0 & 0 & 1 \\
\hline Consumo de arogas & GCD & 0 & 0 & 0 & 0 & 1 \\
\hline Prótese dentária & GSD & 0,09 & 0,3 & 0 & 1 & \\
\hline Protese dentarla & GCD & 0 & 0 & 0 & 0 & 0,306 \\
\hline & GSD & 0,64 & 0,5 & 0 & 1 & \\
\hline Fatores hormonals & GCD & 0,83 & 0,39 & 0 & 1 & 0,304 \\
\hline Fscore final & GSD & 24,36 & 2,01 & 22 & 27 & 0743 \\
\hline Escore tinal & GCD & 34,75 & 3,44 & 19 & 30 & 0,743 \\
\hline
\end{tabular}

GSD: grupo sem disfonia; GCD: grupo com disfonia

a Teste ANOVA.

${ }^{\mathrm{b}} \mathrm{p}<0,05$ 
Quanto às médias das RSR obtidas durante as aulas, verificou-se tendência à diferença entre os grupos ( $p=0,096)$, com média maior para o GCD (Tabela 3). Quando avaliada cada sala de aula separadamente, verificou-se que a RSR estava alterada em nove delas (39\%), considerando a norma para audição normal, e em 23 salas (95\%), considerando a norma para perda auditiva.

Quando avaliados os recursos vocais nas duas diferentes situações (ruído e silêncio), observou-se que, na situação-ruído, um maior número de professores realizou ataque vocal brusco comparativamente à situação-silêncio. Além disso, as professoras mostraram tendência a tornar a articulação dos sons da fala mais precisa na situação-ruído $(p=0,052)$ (Tabela 4).

A partir da relação encontrada entre exposição ao ruído e ataque vocal brusco, realizou-se análise de risco para esse fator. Com isso, identificamos que o risco de realizar ataque vocal brusco na situação-ruído corresponde a 21,7\% (IC95\% 9,2-42,3). No ambiente silencioso, o risco foi inexistente (IC95\% 0-16,9).

Quanto às médias dos níveis de ruído medidos nas salas de aula, não foi identificada diferença entre os grupos para nenhuma medida, ainda que os valores das salas do grupo GCD tenham sido mais elevados. As médias de Leq para ambos os grupos demonstram que o ruído nas salas de aula não está adequado para a maioria delas. Apenas duas salas de aula $(8,7 \%)$ no GSD apresentaram-se com ruído abaixo de $50 \mathrm{~dB}(\mathrm{~A})$ durante as aulas.

Tabela 2. Média, desvio padrão, mínimo e máximo referentes à análise acústica e perceptivo-auditiva para os grupos com e sem disfonia.

\begin{tabular}{|c|c|c|c|c|c|c|}
\hline Característica & Grupo & Média & DP & Mínimo & Máximo & $\mathbf{p}^{\mathrm{a}}$ \\
\hline \multicolumn{7}{|c|}{ Análise medidas acústicas } \\
\hline \multirow{2}{*}{ Frequência fundamental $(\mathrm{Hz})$} & GSD & 191,19 & 22,37 & 164,45 & 231,92 & \multirow{2}{*}{0,702} \\
\hline & GCD & 195,19 & 26,85 & 142,41 & 244,24 & \\
\hline \multirow{2}{*}{ Jitter (\%) } & GSD & 0,39 & 0,21 & 0,21 & 0,95 & \multirow{2}{*}{0,166} \\
\hline & GCD & 0,28 & 0,16 & 0,13 & 0,73 & \\
\hline \multirow{2}{*}{ Shimmer (\%) } & GSD & 4,41 & 1,99 & 1,78 & 7,38 & \multirow{2}{*}{0,794} \\
\hline & GCD & 4,19 & 2,09 & 1,26 & 9,44 & \\
\hline \multirow{2}{*}{ Proporção harmônico-ruído (dB) } & GSD & 18,97 & 5,65 & 10,19 & 27,42 & \multirow{2}{*}{0,889} \\
\hline & GCD & 18,65 & 4,1 & 10,23 & 24,84 & \\
\hline \multicolumn{7}{|c|}{ Análise perceptivo-auditiva CAPE-V } \\
\hline \multirow{2}{*}{ Rugosidade } & GSD & 13,64 & 10,89 & 0 & 34 & \multirow{2}{*}{0,0005} \\
\hline & GCD & 43,42 & 21,42 & 4 & 71 & \\
\hline \multirow{2}{*}{ Soprosidade } & GSD & 19,91 & 9,53 & 5 & 33 & \multirow{2}{*}{$0,0025^{b}$} \\
\hline & GCD & 45,25 & 22,74 & 0 & 73 & \\
\hline \multirow{2}{*}{ Tensão } & GSD & 1,73 & 3,47 & 0 & 11 & \multirow{2}{*}{0,0001} \\
\hline & GCD & 28,5 & 18,83 & 0 & 61 & \\
\hline \multirow{2}{*}{ Pitch } & GSD & 8,73 & 10,08 & 0 & 26 & \multirow{2}{*}{$0,0093^{b}$} \\
\hline & GCD & 27,17 & 19,01 & 0 & 73 & \\
\hline \multirow{2}{*}{ Loudness } & GSD & 1,27 & 4,22 & 0 & 14 & \multirow{2}{*}{0,0229} \\
\hline & GCD & 12,67 & 14,84 & 0 & 37 & \\
\hline
\end{tabular}

GSD: grupo sem disfonia; GCD: grupo com disfonia

a Teste ANOVA.

${ }^{\mathrm{b}} \mathrm{p}<0,05$

Tabela 3. Média, desvio padrão, mínimo e máximo referentes à relação sinal-ruído para os grupos com e sem disfonia.

\begin{tabular}{lcccccc}
\hline Característica & Grupo & Média & DP & Mínimo & Máximo & p* \\
\hline \multirow{2}{*}{ Relação sinal/ruído (dB) } & GSD & 15,1 & 2,83 & 11,1 & 19,9 & 0,096 \\
& GCD & 17,1 & 2,68 & 13,1 & 21,6 & \\
\hline
\end{tabular}

GSD: grupo sem disfonia; GCD: grupo com disfonia

* Teste ANOVA. 
Tabela 4. Número de professores (n) em cada situação (silêncio e ruído) e em cada classificação dos recursos vocais.

\begin{tabular}{|c|c|c|c|c|}
\hline Variável & Classificação & Silêncio (n) & Ruído (n) & $\mathrm{p}^{\mathrm{a}}$ \\
\hline \multirow{2}{*}{ Tipo de voz } & Neutra & 18 & 19 & \multirow{2}{*}{0,729} \\
\hline & Não-neutra, desviada ou alterada & 5 & 4 & \\
\hline \multirow{2}{*}{ Ataque vocal } & Isocrônico & 23 & 18 & \multirow{2}{*}{0,024} \\
\hline & Brusco & 0 & 5 & \\
\hline \multirow{2}{*}{ Loudness } & Médio & 16 & 11 & \multirow{2}{*}{0,15} \\
\hline & Fraco ou forte & 7 & 12 & \\
\hline \multirow{2}{*}{ Pitch } & Médio & 14 & 16 & \multirow{2}{*}{0,155} \\
\hline & Agudo ou grave & 9 & 7 & \\
\hline \multirow{2}{*}{ Ressonância } & Equilibrada & 10 & 13 & \multirow{2}{*}{0,397} \\
\hline & Não equilibrada & 13 & 10 & \\
\hline \multirow{2}{*}{ Articulação } & Precisa & 17 & 22 & \multirow{2}{*}{0,052} \\
\hline & Reduzida & 6 & 1 & \\
\hline \multirow{2}{*}{ Velocidade de fala } & Adequada & 23 & 22 & \multirow{2}{*}{0,5} \\
\hline & Aumentada & 0 & 1 & \\
\hline \multirow{2}{*}{$\begin{array}{l}\text { Coordenação } \\
\text { respiração-fala }\end{array}$} & Presente & 20 & 23 & \multirow{2}{*}{0,116} \\
\hline & Ausente & 3 & 0 & \\
\hline
\end{tabular}

a Teste qui-quadrado

${ }^{\mathrm{b}} \mathrm{p}<0,05$

Tabela 5. Média, desvio padrão referentes aos níveis de ruído (Leq, mínimo e máximo em dBA) para os gruposcom e sem disfonia.

\begin{tabular}{lcccc}
\hline Variável & Grupos & Média & DP & P* \\
\hline \multirow{2}{*}{ Leq } & GSD & 68,91 & 16,63 & 0,180 \\
\multirow{2}{*}{ Máximo } & GCD & 76,22 & 7,32 & \\
& GSD & 80,25 & 23,52 & 0,242 \\
Mínimo & GCD & 88,97 & 8,52 & \\
& GSD & 53,16 & 7,05 & 0,361 \\
\hline
\end{tabular}

GSD: grupo sem disfonia; GCD: grupo com disfonia

* Teste ANOVA.

\section{DISCUSSÃO}

A voz do professor é um tema muito investigado, mas poucos estudos relacionam as questões ambientais ao risco de disfonia ${ }^{7,13}$. Avaliar esse risco constitui ferramenta importante para ações de promoção da saúde no ambiente escolar, que podem nortear um processo de conscientização dos envolvidos, bem como mudanças de hábitos, além de adequações ambientais que favoreçam a melhora da qualidade de vida. Além disso, ações de promoção da saúde nesse ambiente favorecem o diagnóstico mais precoce das disfonias ${ }^{1}$.

O grupo de participantes desta pesquisa foi composto por professoras com média de idade de 37,26 anos. Sabe-se que há maior frequência de mulheres nessa profissão; porém, a faixa etária é bastante variada ${ }^{3,5,7,10,19,22,24, \mathrm{~b}}$. Quando comparadas aos homens, as mulheres se mostram mais propensas a alterações vocais pela própria configuração laríngea ${ }^{13,18}$.

Por meio do PRRD-Geral e do PRRD-Específico, observamos neste estudo que, independentemente de apresentar disfonia, os professores estão expostos a riscos semelhantes para disfonia, como encontrado em estudo anterior ${ }^{\mathrm{b}}$.

Quanto ao PRRD-Geral, houve diferença entre os grupos apenas quanto à prática regular de atividade física, maior no grupo sem disfonia. No grupo sem disfonia, também foi menor a ocorrência de tensão vocal. Praticantes de atividade física podem ter menores níveis de 
estresse e tensão corporal ${ }^{14} \mathrm{e}$, consequentemente, vocal, o que sugere uma relação entre sedentarismo e disfonia ${ }^{1}$. Além disso, a prática de exercícios físicos diminui os sintomas de depressão e ansiedade $\mathrm{e}^{14}$, o que impactaria positivamente para a produção vocal sem tensão e na qualidade de vida dos professores. Ressalta-se que a prática de atividade física depende de rotina e hábitos de vida estabelecidos pelo indivíduo e pode ser reforçada em ações de promoção de saúde.

Em relação ao PRRD-Específico, os grupos diferenciaram-se quanto às particularidades da profissão atual, com o GSD com maior escore. Ao observar mais detalhadamente esses dados, observou-se que, no GSD, havia somente professores considerados regentes e apenas no GCD havia professores especialistas (artes, música e informática). No cálculo do escore, o professor regente recebe um ponto a mais do que o especialista por se considerar que, com muita frequência, o especialista destina parte da carga horária das aulas à prática dos alunos e, por isso, poderia se supor que dá menos tempo de aula expositiva do que o regente, o que seria positivo para o bem-estar vocal. Mas, se todos os professores especialistas estavam no GCD, esse fator pode estar sendo minimizado por outros de maior risco para disfonia. Deve-se considerar ainda que as crianças podem ficar mais agitadas nessas aulas, o que poderia levar o professor especialista a retomar os limites fazendo uso da voz. Estudos com outras escolas irão possibilitar análises mais amplas e que poderão indicar, inclusive, a necessidade de revisão do cálculo do escore do PRRD-Específico.

Outro aspecto desse subitem que pontuou mais no GSD foi o número de anos de atuação das professoras junto ao nível de ensino atual. Contrariamente ao encontrado na literatura ${ }^{15,25}$, as professoras sem alteração vocal indicaram mais anos lecionando em sala de aula em comparação às professoras do GCD. Esse dado também foi observado em outro estudo ${ }^{7}$, na caracterização da amostra, em que apenas $33 \%$ dos professores com distúrbio vocal apresentavam mais de dez anos de atuação. Esse achado pode indicar uma adaptação positiva dessas profissionais ao longo do tempo ou mesmo algum treinamento recebido, fatores que também podem ser melhor explorados em estudos futuros. Em relação ao nível de ensino, a maior parte das professoras do GSD lecionam para o ensino fundamental e metade das professoras do GCD atuam junto à educação infantil. Estudo realizado com professoras da educação infantil ${ }^{23}$ encontrou prevalência de disfonia mais elevada em comparação a outras pesquisas com professores do ensino fundamental, médio e superior, o que pode indicar que as particularidades do trabalho junto a essa faixa etária se configuram como mais um fator a ser considerado.

Desse modo, levando-se em conta as particularidades do trabalho, devem ser consideradas as modificações que podem ocorrer a cada semestre na grade dos docentes quanto às horas/aula e que justificariam um monitoramento periódico para avaliação de possíveis tendências de aumento ou diminuição de riscos; além disso, esse controle poderia contribuir com análises acerca de possível fadiga vocal ao final de cada semestre.

Embora não tenham sido observadas diferenças entre os grupos no que se refere ao escore final do PRRD-Geral e do PRRD-Específico, observou-se maior média no escore do GCD, o que poderia ser melhor evidenciado em estudos com amostras maiores.

As médias obtidas no escore do PRRD-Geral para ambos os grupos se encontraram acima da nota de corte para mulheres, que é de $29,25^{15}$. Isso representa elevado risco para disfonia para as professoras com e sem alteração vocal, corroborando outros estudos ${ }^{1, b}$.

A diminuição de riscos requer mudanças nas condições e organização do trabalho e depende de mobilizações de instâncias maiores ${ }^{5}$. A partir deste estudo, observou-se que, independentemente da presença e do grau de alteração vocal, os riscos ocupacionais para disfonia aos quais os professores se expõem diariamente são elevados ${ }^{1,7, b}$.

No que diz respeito à análise acústica das vozes gravadas em ambiente silencioso, de acordo com os parâmetros avaliados, não foram encontradas diferenças entre os grupos. Em estudo pregresso com 28 educadoras, com análise vocal acústica realizada por meio do 
mesmo software, foram comparados grupos de professores com vozes adequadas, vozes alteradas em grau leve e alteradas em grau moderado ${ }^{24}$. Assim como nesta pesquisa, os autores $^{24}$ não observaram diferenças para os parâmetros acústicos frequência fundamental, shimmer e proporção harmônico-ruído. No entanto, encontraram diferenças quanto ao jitter. No presente estudo, apesar de não haver diferença entre os grupos em relação aos parâmetros vocais na análise acústica, a média encontrada no GCD está abaixo dos valores de referência. Pesquisa com 99 professoras, com análise acústica das vozes feita por meio de outro software (Multi-Dimensional Voice Program Advanced), encontrou medidas de jitter, shimmer e frequência fundamental acima da normalidade ${ }^{21}$. Os valores da proporção harmônico-ruído foram abaixo do esperado nos dois grupos, indicando que, no GSD, as vozes consideradas sem alteração estão apresentando mais ruído do que seria esperado, o que pode ser sinal de fadiga vocal ${ }^{24}$. Esse dado mostra a importância de se combinar a análise perceptivo-auditiva e a acústica para maior detalhamento.

Na análise vocal perceptivo-auditiva pelo CAPE-V, foram encontradas diferenças em todos os aspectos avaliados quando comparados os dois grupos, com desvios no GCD. Ainda assim, o GSD apresentou soprosidade e rugosidade, ainda que reduzida e não configurando disfonia, mas que pode estar associada às alterações observadas em medidas acústicas como jitter e proporção harmônico-ruído discutidas anteriormente.

A presença maior de rugosidade nas professoras com disfonia pode estar relacionada a alterações de massa nas pregas vocais, como nódulos vocais que são comumente encontrados nessa categoria profissiona ${ }^{22} \mathrm{e}$ mais frequentemente em mulheres, assim como a soprosidade, por causa da própria configuração laríngea, propensa à existência de fenda glótica ${ }^{18}$. Estes dados enfatizam a importância de avaliação das condições laríngeas das professoras com disfonia.

As alterações vocais encontradas reforçam os achados de outros estudos ${ }^{21,22}$ e justificam a realização de ações de promoção de saúde e bem-estar vocal que melhorem a qualidade de voz desses profissionais. Intervenções simples, como prática de aquecimento vocal e treino respiratório, apresentam resultados positivos ${ }^{16}$.

No que concerne à RSR, houve tendência de média mais elevada no GCD, sugerindo que, nas salas dos professores com disfonia, a voz seja mais intensa do que nas demais. Esse dado concorda com outros estudos ${ }^{20,24}$ e representa fator de risco ocupacional importante para esses profissionais.

Na situação-ruído, observou-se a utilização de ataque vocal brusco, com risco de 21,7\% de sua realização durante as aulas. $O$ ataque vocal brusco consiste em um padrão vocal inadequado, relacionado ao fonotrauma, em que ocorre rápida e completa adução das pregas vocais ao início da fonação. Esse estado pode ser acompanhado de tensão muscular e, quando frequente, sinaliza esforço aumentado ${ }^{2}$. Para se fazer entender nas salas de aula, o professor pode alterar seu padrão vocal habitual, nem sempre com ajuste adequado para suas condições laríngeas, como é o caso do ataque vocal brusco ${ }^{20,24}$.

Um fator de proteção observado nos dois grupos foi a tendência a articular os sons da fala de maneira mais precisa na situação de ruído. Além disso, os dois grupos mantiveram adequados pitch, velocidade de fala e coordenação respiração-fala quando expostos à situação de ruído. Ambos resultados sugerem um ajuste positivo natural ou resultado de treinamento prévio.

Dentre os riscos ocupacionais possíveis, o presente estudo enfatizou a investigação dos níveis de ruído presentes em sala de aula, bem como a análise da relação sinal-ruído. O monitoramento dessas duas medidas no contexto escolar é fundamental para guiar ações que minimizem prejuízos para a voz do professor. Além disso, esses níveis, quando acima do recomendado, podem interferir não só no desempenho dos professores, mas também no aprendizado dos alunos ${ }^{11}$.

Com relação ao ruído presente em sala de aula, não houve diferença entre os grupos para as médias de ruído equivalente, ainda que os valores das salas do GCD tenham sido maiores. Além disso, 
apenas duas salas de aula (8,7\%) no GSD apresentaram ruído abaixo do proposto durante as aulas $^{7 . a}$. A relação sinal-ruído foi inferior ao recomendado para crianças com audição normal ${ }^{\mathrm{e}}$ em aproximadamente $39 \%$ das salas, e para crianças com perda auditiva ${ }^{f}$, em aproximadamente 95\%. Os níveis elevados de ruído encontrados reforçam a atual situação das escolas no Brasil ${ }^{7,11,13}$.

Com a redução dos níveis de ruído nas salas de aula, a relação sinal-ruído aumenta sem que o professor precise realizar ajustes no padrão vocal, o que facilita a compreensão dos alunos e diminui o risco de disfonia.

Ressalta-se a necessidade de novas pesquisas que investiguem e enfatizem a importância da relação sinal-ruído também no monitoramento dos problemas vocais, visto que a relação sinal-ruído pode ser importante aliada para controle da intensidade do ruído e situação de escuta oferecidas nas salas de aula.

Programas direcionados nesse sentido poderiam viabilizar medidas preventivas, bem como o desenvolvimento de estratégias que capacitariam os professores e funcionários da escola como agentes promotores de saúde.

\section{REFERÊNCIAS}

1. Bassi IB, Assunção AA, Gama ACC, Gonçalves LG. Características clínicas, sócio demográficas e ocupacionais de professoras com disfonia. Disturb Comun. 2011 [citado 30 set 2017];23(2):173-80. Disponível em: https://revistas.pucsp.br/index.php/dic/article/view/8273

2. Behlau M, Madazio G, Feijó D, Pontes P. Avaliação da voz. In: Behlau M, organizadora. Voz: o livro do especialista. Rio de Janeiro: Revinter; 2001. v. 1, p.91-113.

3. Behlau M, Zambon F, Guerrieri AC, Roy N. Epidemiology of voice disorders in teachers and nonteachers in Brazil: prevalence and adverse effects. J Voice. 2012;26(5):665.e9-18. https://doi.org/10.1016/j.jvoice.2011.09.010

4. Bentler RA. List equivalency and test-retest reliability of the speech in noise test. Am J Audiol. 2000;9(2):84-100. https://doi.org/10.1044/1059-0889(2000/010)

5. Biserra MP, Giannini SPP, Paparelli R, Ferreira LP. Voz e trabalho: estudo dos condicionantes das mudanças a partir do discurso de docentes. Saude Soc. 2014;23(3):966-78. https://doi.org/10.1590/S0104-12902014000300019

6. Chen SH, Chiang SC, Chung YM, Hsiao LC, Hsiao TY. Risk factors and effects of voice problems for teachers. J Voice. 2010;24(2):183-92. https://doi.org/10.1016/j.jvoice.2008.07.008

7. Cutiva LC, Vogel I, Burdorf A. Voice disorders in teachers and their associations with work-related factors: a systematic review. J Commun Disord. 2013;46(2):143-55. https://doi.org/10.1016/j.jcomdis.2013.01.001

8. Dreossi RCF, Momensohn-Santos T. Noise and its interference over students in a classroom environment: literature review. Pro-Fono Rev Atual Cient. 2005;17(2):251-8. https://doi.org/10.1590/S0104-56872005000200014

9. Feijó D. Avaliando a comunicação oral. In: Kyrillos LR, organizadora. Fonoaudiologia e telejornalismo. São Paulo: Revinter; 2003. p.75-88.

10. Houtte V, Claeys S, Wuyts F, Van Lierde KV. The impact of voice disorders among teachers: vocal complaints, treatment-seeking behavior, knowledge of vocal care, and voice-related absenteeism. / Voice. 2011;25(5):571-5. https://doi.org/10.1016/j.jvoice.2010.04.008

11. Libardi A, Gonçalves CGO, Vieira TPG, Silverio KCA, Rossi D, Penteado RZ. O ruído em sala de aula e a percepção dos professores de uma escola de ensino fundamental de Piracicaba. Disturb Comun. 2006 [citado 30 set 2017];18(2):167-78. Disponível em: https://revistas.pucsp.br/index. php/dic/article/view/11782

12. Martins PC, Couto TE, Gama ACC. Avaliação perceptivo-auditiva do grau de desvio vocal: correlação entre escala visual analógica e escala numérica. CoDAS. 2015;27(3):279-84. https://doi.org/10.1590/2317-1782/20152014167

13. Medeiros AM, Barreto SM, Assunção AA. Voice disorders (dysphonia) in public school female teachers working in Belo Horizonte: prevalence and associated factors. I Voice. 2008;22(6):676-87. https://doi.org/10.1016/j.jvoice.2007.03.008 
14. Mello MT, Lemos VA, Antunes HKM, Bittencourt L, Santos-Silva R, Tufik S. Relationship between physical activity and depression and anxiety symptoms: a population study. I Affect Disord. 2013;149(1-3):241-6.https://doi.org/10.1016/j.jad.2013.01.035

15. Nemr K, Simões-Zenari M, Duarte JMT, Lobrigate KE, Bagatini FA. Dysphonia risk screening protocol. Clinics. 2016;71(3):114-27. https://doi.org/10.6061/clinics/2016(03)01

16. Pereira LPP, Masson MLV, Carvalho FM. Aquecimento vocal e treino respiratório em professores: ensaio clínico randomizado. Rev Saude Publica. 2015;49:67. https://doi.org/10.1590/S0034-8910.2015049005716

17. Pinho SMR, Tsuji DH. Fundamentos em laringologia e voz. Rio de Janeiro: Revinter; 2006.

18. Pontes P, Kyrillos L, Behlau M, De Biase N, Pontes A. Vocal nodules and laryngeal morphology. J Voice. 2002;16(3):408-14. https://doi.org/10.1016/S0892-1997(02)00112-1

19. Provenzano LCFA, Sampaio TMM. Prevalência de disfonia em professores do ensino púbico estadual afastados de sala de aula. Rev CEFAC. 2010;12(1):97-108. https://doi.org/10.1590/S1516-18462010000100013

20. Rantala LM, Hakala S, Holmqvist S, Sala E. Classroom noise and teacher's voice production. J Speech Lang Hear Res. 2015;58(5):1397-406. https://doi.org/10.1044/2015_JSLHR-S-14-0248

21. Ribeiro VV, Cielo CA. Medidas vocais perceptivo-auditivas e acústicas, queixas vocais e características profissionais de professoras de Santa Maria (RS). Audiol Commun Res. 2014;19(4):387-98. https://doi.org/10.1590/S2317-64312014000400001395

22. Servilha EAM, Correia JM. Correlações entre condições do ambiente, organização do trabalho, sintomas vocais autorreferidos por professores universitários e avaliação fonoaudiológica. Disturb Comun. 2014 [citado 30 set 2017];26(3):452-62. Disponível em: https://revistas.pucsp. br/index.php/dic/article/view/16222

23. Simões M, Latorre MRDO. Prevalência de alteração vocal em educadoras e sua relação com a auto-percepção. Rev Saude Publica. 2006;40(6):1013-8. https://doi.org/10.1590/S0034-89102006000700008

24. Simões-Zenari M, Bitar ML, Nemr NK. Efeito do ruído na voz de educadoras de instituições de educação infantil. Rev Saude Publica. 2012;46(4):657-64. https://doi.org/10.1590/S0034-89102012005000038

25. Souza CL, Carvalho FM, Araújo TM, Reis EJFB, Lima VMC, Porto LA. Fatores associados a patologias de pregas vocais em professores. Rev Saude Publica. 2011;45(5):914-21. https://doi.org/10.1590/S0034-89102011005000055

Contribuição dos Autores: Concepção e planejamento do estudo, análise e interpretação dos dados, elaboração da redação e revisão do manuscrito, aprovação da versão final a ser publicada e responsabilidade pública pelo conteúdo do artigo: BGS, TVS, MSZ, RRM, AGS, KN. Coleta dos dados: BGS, TVS. Orientação quanto à elaboração da redação e coleta dos dados: MSZ, RRM, AGS, KN.

Conflito de Interesses: Os autores declaram não haver conflito de interesses. 